\title{
On the reduction of the third-order harmonic losses in low-voltage power cables used for feeding large LED and CFL lighting loads
}

\author{
N. Milardovich ${ }^{1, *}$, L. Prevosto ${ }^{2}$, M. A. Lara ${ }^{3}$ and D. Milardovich ${ }^{4}$ \\ ${ }^{1}$ Laboratorio de Electricidad, Departamento Ingeniería Electromecánica \\ Facultad Regional Venado Tuerto (UTN) Laprida 651 (2600), Venado Tuerto (Santa Fe), Argentina \\ ${ }^{2}$ Grupo de Descargas Eléctricas, Departamento Ingeniería Electromecánica \\ Facultad Regional Venado Tuerto (UTN) Laprida 651 (2600), Venado Tuerto (Santa Fe), Argentina \\ ${ }^{3}$ Facultad de Ciencias Exactas, Ing. y Agrimensura. Maestría en Energía para el Desarrollo Sostenible (UNR). Av. \\ Pellegrini \\ 250 (2000), Rosario (Santa Fe), Argentina \\ ${ }^{4}$ Facultad de Ciencias Exactas, Ing. y Agrimensura (UNR). Av. Pellegrini 250 (2000), Rosario (Santa Fe), Argentina \\ *corresponding author, E-mail: nmilardovich@gmail.com
}

\begin{abstract}
An experimental investigation of diversity factors for LED (light emitting diode) in combination with CFL (compact fluorescent lamps) and LED lamps is presented in this paper. Attention was paid to the reduction of low-order harmonic currents, especially the third one; which is mainly responsible for the strong increase in power losses in the neutral conductor of low-voltage installations. The harmonic currents drawn by several LED and CFL lamps with nominal powers $<25 \mathrm{~W}$ were first measured to investigate the electrical characteristics of individual lamps. The results showed a sensible reduction of the harmonic current of third order and therefore a marked reduction of the power losses associated with them. The convenience of having LED lamps designed to operate as two-phase loads is suggested for certain applications of significant power demand.
\end{abstract}

\section{Introduction}

Due to global government policies, incandescent lamps were gradually replaced by compact fluorescent lamps (CFL), and the latter are now being replaced by light emitting diodes (LED) [1-3], seeking a reduction in lighting costs and a lower impact on environment.

Although LED technology usually shows greater efficiency compared to older lighting technologies, it usually introduces harmonic currents of relatively large amplitude, especially of third order, in the electrical networks destined to these applications $[4,5]$. In general lighting applications a compact ac/dc (alternating current/direct current) converter [6-8] should be used to supply dc current to LEDs, which introduce nonlinearity to the system. As nonlinear loads, LEDs might produce highly-distorted currents. These distorted currents are injected into the power system thus producing a large number of problems, such as additional power losses in conductors, which are dependent on the harmonic frequency [9-11].

A frequency domain analysis [4] of the harmonic currents $\left(I_{p u}(h)\right)$ produced by several commerciallyavailable LEDs (with power ranging 3 to $120 \mathrm{~W}$ ) is presented in Fig. 1. $I_{p u}(h)$ was expressed in per-unit of the fundamental harmonic current $(h=1$ corresponding to a harmonic frequency of $f=50 \mathrm{~Hz}$, being $h$ the order of the harmonic). It is observed that the amplitude of the harmonic currents decreases almost inversely with the order of the harmonic; thus indicating that the third-order one is usually the most significant. FCLs also tend to present considerable amplitudes in their third-order harmonics [12].

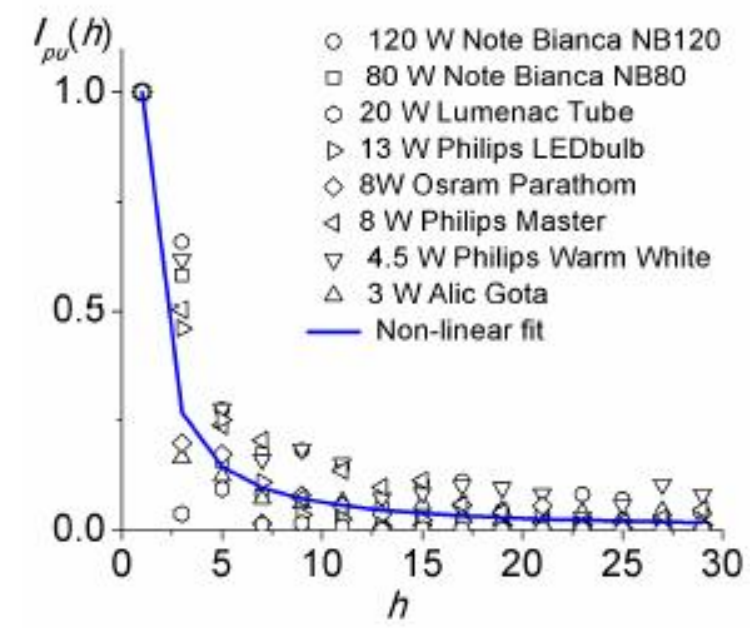

Figure 1: Harmonic currents content of different LED lamps. Taken from [4].

In four-core cables (three phases and neutral) the current in the neutral conductor may be larger than the current in the phase conductors when significant third-order harmonic currents are present $[4,9,10]$. This fact may lead to overheating of the neutral conductor unless the neutral is properly sized. Since several national standards allow the neutral conductor to have a reduced cross-section with respect to the phase conductors section (about half of it), a large number of the existing low-voltage installations present a section reduction in the neutral conductor [13]. These installations when feeding non-linear LED and FCL 
loads could present more than twice the losses corresponding to a current without distortion of the same effective value (RMS) as the value of the first harmonic current of the lamp [4].

A large number of research work was done on LED lamps as an energy-efficient lamp, but most of them have been dedicated to design the circuit that links the network to the LED [14,15]. Several other works have focused on the light distribution and visual performance of LED lamps (e.g., [16]), however, a relatively small amount of works focused on the harmonic emissions of LED and CFL lamps and in strategies to reduce the harmonic distortion problem generated by them $[12,17]$.

A research on the diversity factor for combinations of LED lamps with powers in the range 3 to $8 \mathrm{~W}$ was presented in [17]. It was found that the diversity factor becomes smaller if a large number of lamps are combined. However, such a reduction was more significant for high-order harmonic currents. In particular a diversity factor close to one for the third-order harmonic was found for the combinations studied. In [12] an investigation was presented on the diversity factor for combinations of LED and FCL lamps. The measurements showed a considerable reduction of the diversity factor for higher harmonic currents but, on the other hand, the low-order harmonics did not show a significant decrease.

In this paper we present a research on the diversity factor with combinations of LED and FCL lamps with powers in the range of 3-23 W. The harmonic emission of the lamps was also studied. Attention was paid to the reduction of low-order harmonics, especially the third one; which is mainly responsible for the strong increase in power losses in the neutral conductor of the low-voltage installations [4]. The results showed that it is possible to significantly reduce the diversity factor for the third harmonic by appropriately combining LED and FCL lamps.

\section{Experimental method \\ 2.1. Experimental arrangement}

The experimental set-up used to evaluate the diversity factor and the content of harmonic currents in different combinations of LED and CFL lamps are shown in Fig. 2.

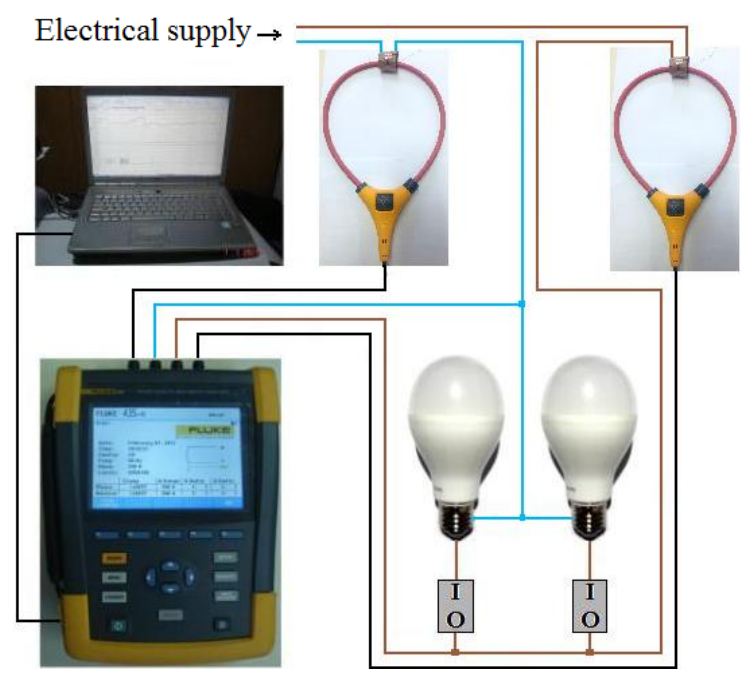

Figure 2: Experimental setup for harmonic measurements.

A power quality analyzer (Fluke 435-II) was used in the measurements. In order to match the measuring range of the instrument used to the relatively small currents of the lamp combinations, the currents were measured by using lowinductance $(\sim 0.1 \mathrm{mH})$ coils. It was verified during the measurements that the inductance introduced in the circuit by the coils does not appreciably affects the results. The uncertainty $( \pm 2 \%)$ in the current measurement due to the position sensitivity of the used flexible current probe was accounted for. The information provided by the instrument was processed through the Powerlog 4.3.1 software.

Technical data for tested LED and CFL lamps, broadly used in general lighting applications with powers varying between 3 and $23 \mathrm{~W}$; are given in Table I.

\begin{tabular}{|c|c|c|c|}
\hline $\begin{array}{c}\text { Type of } \\
\text { lamp } \\
\text { (no.) }\end{array}$ & $\begin{array}{c}\text { Trade } \\
\text { name }\end{array}$ & Model/Code & $\begin{array}{c}\text { Nominal } \\
\text { power } \\
\text { (W) }\end{array}$ \\
\hline LED1 & Alic & 7798118819944 & 3 \\
\hline LED2 & Silvania & GLS A 55 Toledo & 5.5 \\
\hline LED3 & Osram & Parathom & 8 \\
\hline LED4 & Philips & 8F27A60 & 8 \\
\hline LED5 & Lumenac & A-60-8/830 & 8 \\
\hline LED6 & Sica & 911529 & 9 \\
\hline LED7 & Philips & $9290002497 \mathrm{C}$ & 9 \\
\hline LED8 & GE & 7501011856073 & 10 \\
\hline LED9 & Philips & 9290002500 & 13 \\
\hline LED10 & Philips & 9290011639 & 13 \\
\hline LED11 & Sica & 7791772048880 & 13 \\
\hline LED12 & Philips & $9290002777 \mathrm{C}$ & 14 \\
\hline LED13 & TBCin & A60-SMD-14W & 14 \\
\hline LED14 & Sica & 911593 & 15 \\
\hline LED15 & Philips & $9290011650 \mathrm{~A}$ & 18 \\
\hline
\end{tabular}




\begin{tabular}{|c|c|c|c|}
\hline CFL1 & Sica & 914178 & 11 \\
\hline CFL2 & Osram & $\begin{array}{c}\text { Duluxstar Mini } \\
\text { Twister }\end{array}$ & 13 \\
\hline CFL3 & Philips & 8710163405421 & 15 \\
\hline CFL4 & Silvania & 8710163405421 & 20 \\
\hline CFL5 & Philips & Mini Twister WW & 23 \\
\hline
\end{tabular}

Table 1: Technical data for tested lamps.

\subsection{Experimental results and discussion}

Figure 3 shows the amplitude of the third harmonic current (expressed in per-unit of the fundamental harmonic) of the LEDs and CFLs given in Table I. The red line (representing a constant value of 0.86 ) indicates one of the criteria established by IEC 61000-3-2 for the harmonic emission limits for lamps having an active input power $\leq 25 \mathrm{~W}$ (i.e., that the third harmonic current should not exceed $86 \%$ of the fundamental one).

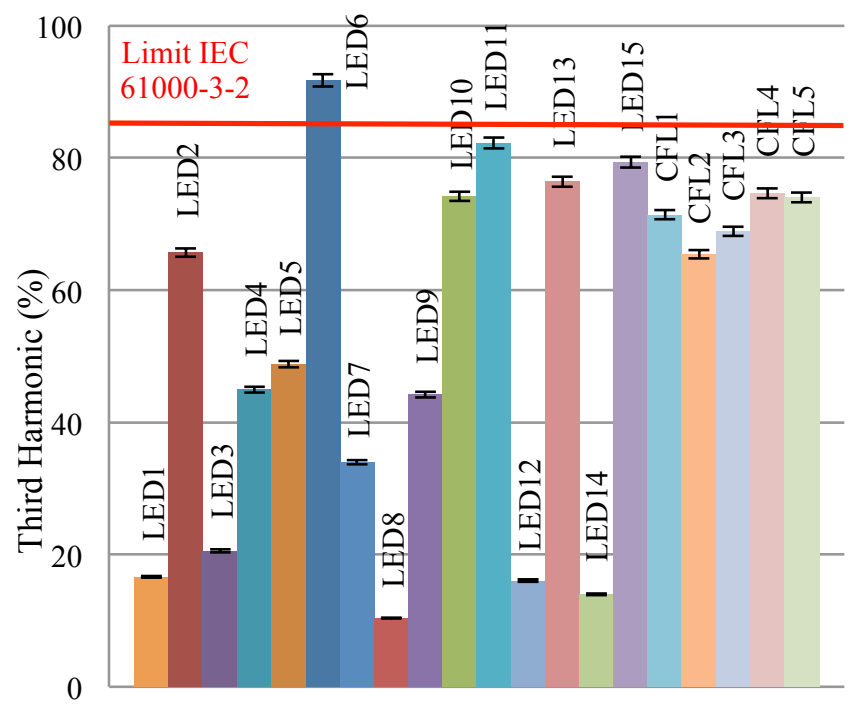

Figure 3: Third-order harmonic current amplitude for the lamps used.

As it can be seen in Fig. 3, the lamps tested meet the quoted emission limit imposed by IEC, except one of them (LED6), which is slightly above it.

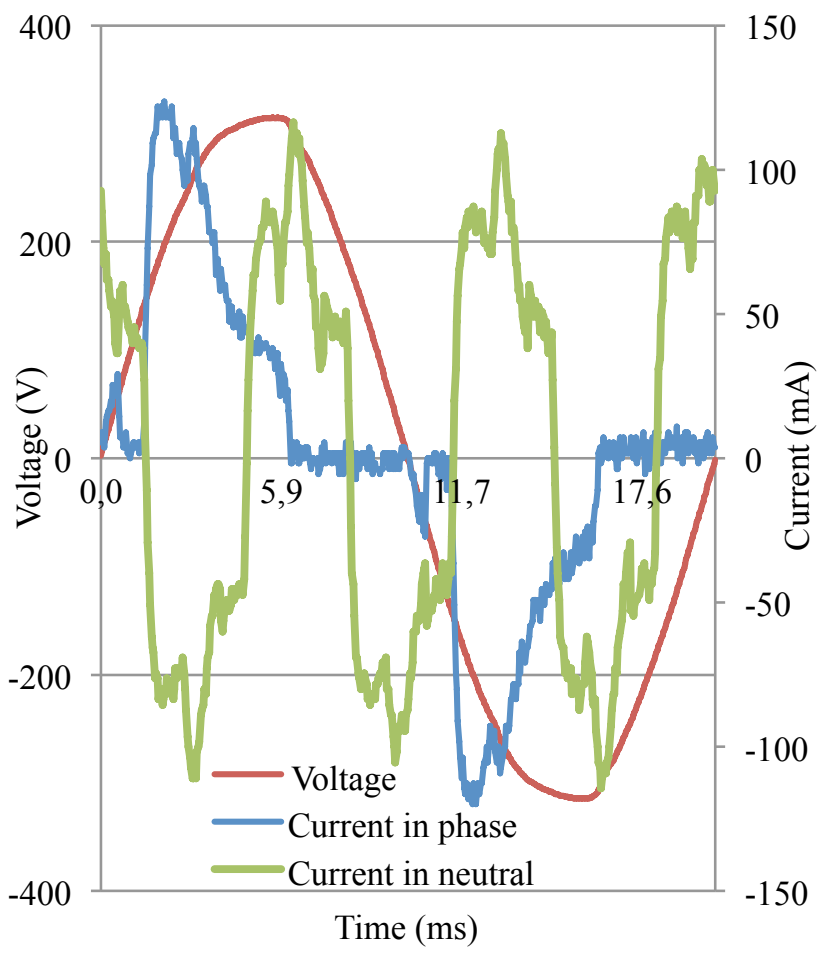

Figure 4: Voltage and current waveforms in one phase and current in the neutral.

Figure 4 shows the current in one phase and in the neutral conductor of a four-core cable that feeds a balanced load of three identical LED (one per phase). Specifically, Fig. 4 was obtained by testing LED lamps identified in Table 1 as LED4. It can be seen that the LED current is highly distorted with respect to a sinusoidal waveform, and that the fundamental frequency of the current in the neutral conductor is $150 \mathrm{~Hz}$ (i.e., the frequency of the third harmonic). Because the third-order harmonic currents (and their multiples) are zero-sequence, they are added at the neutral conductor. All experiments were done with an almost sinusoidal voltage waveform. The total harmonic distortion (THD) of the phase voltage waveform was relatively low $(<3 \%)$.

LED and CFL lamps from different manufacturers have different operating parameters. These differences in the operating parameters are due in part to the different topologies of the compact ac/dc converters used by manufacturers. In particular, diversity of topologies causes a diversity of phase angles and amplitudes of harmonic currents generated by these lamps. This leads to a lower vector sum than the arithmetical sum (which neglects phase angles) when different types of lamps are present, as described in $[12,17]$.

In order to reduce the third-order harmonic currents by properly combining lamps that have an important phase difference in their corresponding harmonic currents (rather than combining lamps in a random way); the phase angle $\left(\varphi_{3}\right)$ of the third-order harmonic currents was first measured (with respect to the fundamental harmonic voltage angle of the phase 1) for the CFL and LED lamps. The results are given in Table 2. Data in Table 2 correspond to time- 
averaged values over an interval of 5 minutes with a sampling rate of $1 \mathrm{~S} / \mathrm{s}$. The data were taken applying nominal voltage to the lamps once they acquired their stable working temperature.

\begin{tabular}{|c|c|c|c|}
\hline $\begin{array}{c}\text { Lamp } \\
\text { no. }\end{array}$ & $\begin{array}{c}\varphi_{3} \\
\text { (degrees) }\end{array}$ & $\begin{array}{c}\text { Lamp } \\
\text { no. }\end{array}$ & $\begin{array}{c}\varphi_{3} \\
\text { (degrees) }\end{array}$ \\
\hline LED1 & 95 & LED11 & -129 \\
\hline LED2 & -112 & LED12 & -33 \\
\hline LED3 & -126 & LED13 & -126 \\
\hline LED4 & -77 & LED14 & -130 \\
\hline LED5 & -9 & LED15 & -125 \\
\hline LED6 & -153 & CFL1 & -114 \\
\hline LED7 & -9 & CFL2 & -111 \\
\hline LED8 & -49 & CFL3 & -111 \\
\hline LED9 & 2 & CFL4 & -119 \\
\hline LED10 & -126 & CFL5 & -108 \\
\hline
\end{tabular}

Table 2: Angle of the third-order harmonic current of the lamps used.

During the measurements, the phase angles of the thirdorder harmonic currents were remarkably stable as shows Fig. 5. The corresponding experimental uncertainty (mainly due to statistical fluctuations) was within \pm 5 degrees.

In order to achieve a considerable attenuation effect of the third-order harmonic currents for a given combination of lamps, they not only should fulfill with the condition that its third-order harmonic be strongly out of phase (as is the case of LED1 and LED4 in Table 2), but in addition the amplitudes of each harmonic current should be similar; i.e., the ratio between the amplitude of the third-order harmonic current of each lamp should be approximately unity.

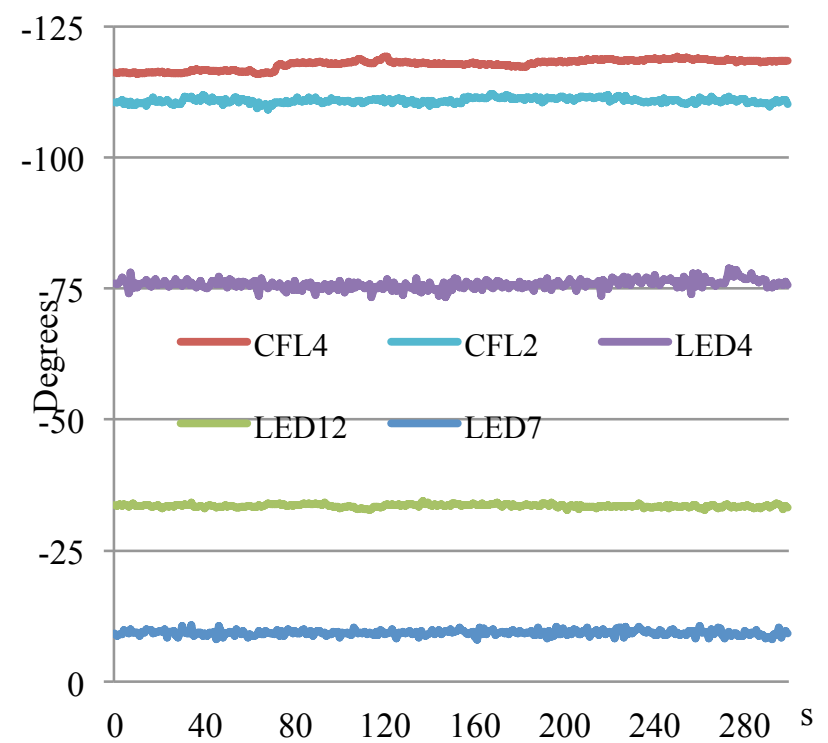

Figure 5: Stability of the phase angle of the third-order harmonic current for several lamps used in this work.
Table 3 shows the results of the ratio between the amplitudes of the third-order harmonic currents for several selected combinations type LED-LED and LED-CFL of the lamps used in this work. The corresponding experimental uncertainty (mainly due to statistical fluctuations) was within $\pm 7 \%$.

\begin{tabular}{|l|c|}
\hline \multicolumn{1}{|c|}{$\begin{array}{c}\text { Lamps } \\
\text { combination }\end{array}$} & $\begin{array}{c}\text { Ratio between the amplitudes (rms) } \\
\text { of the third-order harmonic current } \\
\text { of each lamp of the combination. }\end{array}$ \\
\hline CFL5-LED15 & 1.1 \\
\hline LED10-LED11 & 1.1 \\
\hline CFL3-LED9 & 1.8 \\
\hline LED5-LED6 & 1.9 \\
\hline LED9-LED11 & 1.9 \\
\hline LED3-LED5 & 2.3 \\
\hline CFL1-LED7 & 2.6 \\
\hline LED6-LED7 & 2.8 \\
\hline CFL1-LED3 & 4.6 \\
\hline CFL3-LED12 & 4.7 \\
\hline LED12-LED13 & 4.7 \\
\hline CFL1-LED8 & 6.7 \\
\hline CFL4-LED12 & 6.8 \\
\hline
\end{tabular}

Table 3: Tested combinations of lamps.

To found the level of attenuation of the third-order harmonic currents as a consequence of the combinations of lamps, it is useful to define the diversity factor for the harmonic current, as the ratio between the vector sum (as measured) and the arithmetical sum (as calculated) of the third-order harmonic currents,

$$
D F_{3} \equiv \frac{\mid \text { vector sum of current harmonic } \mid}{\text { arithmetic sum of current harmonic }}
$$

Note that a value of $D F_{3} \approx 1$ indicates an inadequate combination of lamps, which generates a minimum attenuation of the third-order harmonic currents, whereas $D F_{3}<1$ implies an optimum combination, with a maximum attenuation of this harmonic current.

Figure 6 shows the dependence of measured $D F_{3}$ for selected lamp combinations on the difference between the corresponding phase angles of the third-order harmonic currents. The solid curves represent the diversity factor calculated for limit values of the ratio between the amplitudes of the third-order harmonic current of each lamp of the combination (values calculated for a ratio $=1$ are indicated with a blue line while for a ratio $=7$ with a red line). Note that these values are close to the minimum and maximum ratios obtained in the different combinations proposed, as it was indicated in Table 3. 


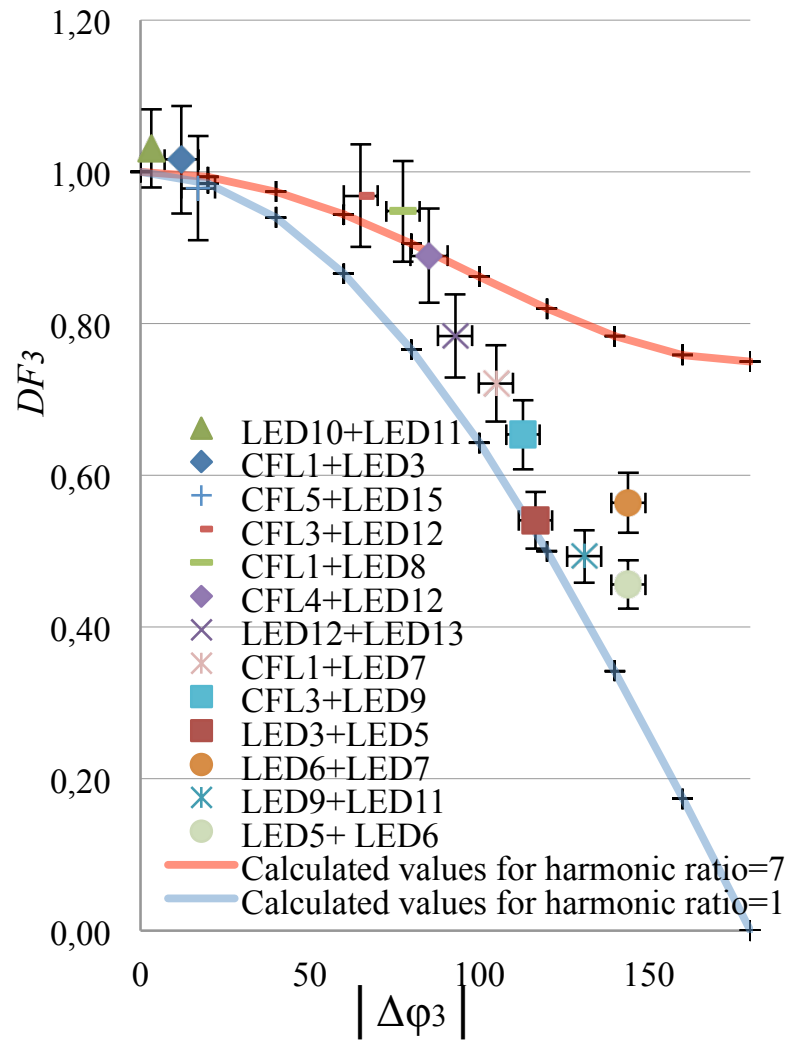

Figure 6: Attenuation of the third harmonic by combination of lamps. See text for details.

It is seen in Fig. 6 that a number of combination of LED and CFL lamps lead to a considerably decrease in the content of the third-order harmonic current. As expected, the maximum attenuation of the third harmonic amplitude is achieved with harmonic ratios close to 1 and for harmonic phase angle differences close to $180^{\circ}$. These results are different from those reported by other studies [12,17] where lower order harmonics did not exhibit a very large reduction in amplitude. However, it should be noted that in $[12,17]$ random combinations of lamps were used.

Note that the results presented in Fig. 6 were obtained combining two lamps; however, the same results could be obtained between two arbitrary sets of lamps, provided that each set is formed by the same number of elements.

Currently CFLs are being replaced by LEDs gradually and in several lighting installations, the two technologies coexist. From the point of view of the reduction of the third harmonic, the combinations of lamps of different technologies are usually convenient. For the lamps evaluated (see Table 1), the change of technology (CFL to LED), not only improves the level of illumination and reduces the content of third harmonic, but also decreases the active power demanded by the installation and, reducing also the environmental impact.

In the case of lighting applications of significant power demand, the case this paper is dedicated to, the single-phase lighting loads are typically powered by four-core cable networks and the loads are distributed as evenly as is possible between the phases. In these large lighting applications the effect of the unbalances due to small asymmetries in the lighting loads distribution or random breakage of some lamps cannot significantly affect the harmonic current regime.

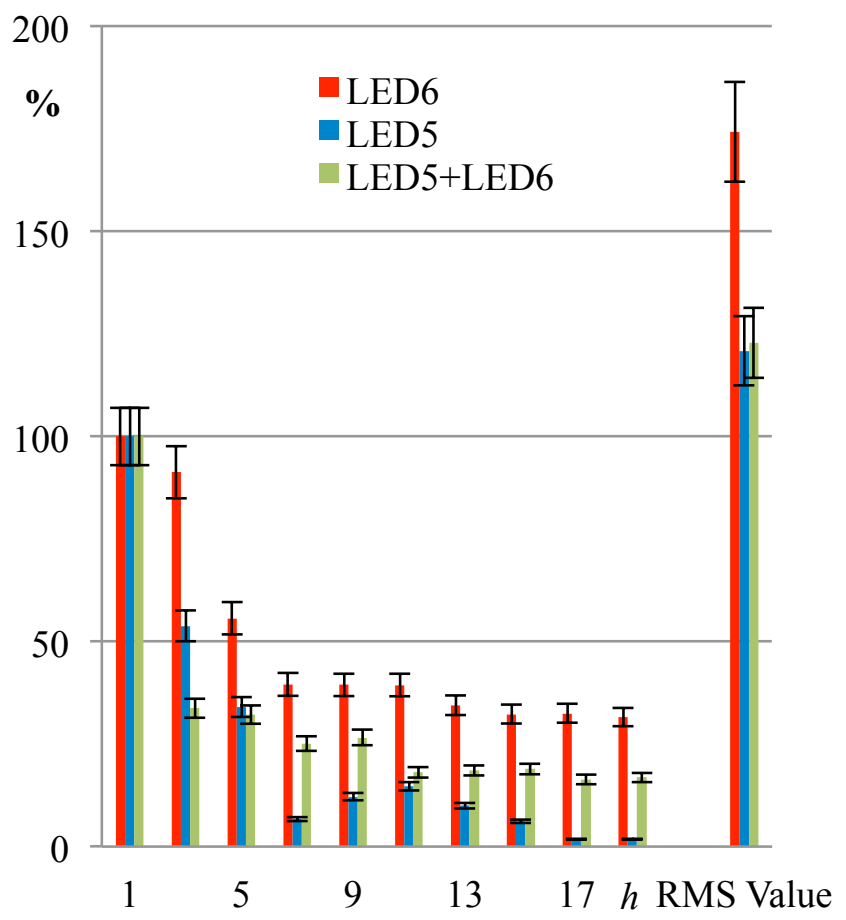

Figure 7: Harmonic currents content in one phase conductor of a four-core cable for a selected LED-LED combination.

In order to better show the contribution of the proposed solution, the measured spectrum of the harmonic currents (expressed in per-unit of the fundamental harmonic current), both in one phase and in the neutral conductor of a four-core cable, feeding balanced single-phase loads formed by selected LED-LED combination is shown in Fig. 7 and 8, respectively. The corresponding spectrums for the individual lamps are also shown. Harmonic currents up to the order $h=$ 19 were measured. In addition, the RMS value of the total current in both conductors is presented in Fig. 7 and 8 .

It is seen in Fig. 7 that the selected LED combination leads to a significant decrease in the third-order harmonic content of the line current. Notice also that the RMS value of the total current of the combination is considerable lower than the corresponding value of the individual lamp having the higher harmonic content of the combination (LED6), being similar to that of the LED5. 


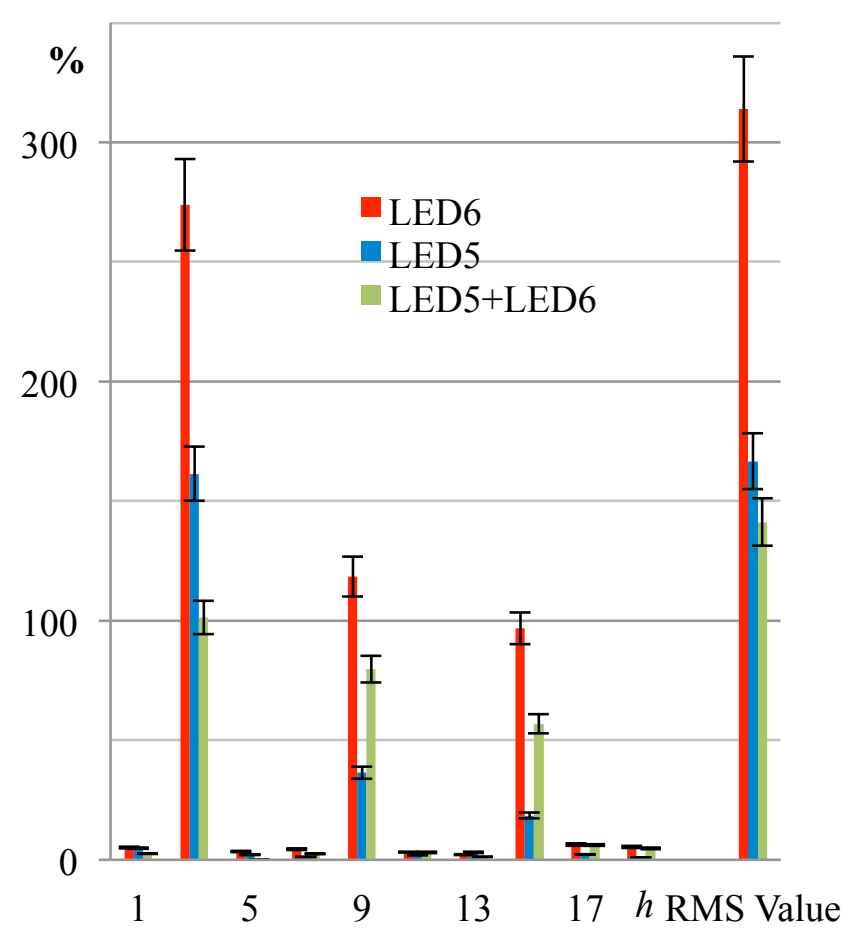

Figure 8: Harmonic currents content in the neutral conductor of a four-core cable for a selected LED-LED combination.

As it can be seen in Fig. 8, the RMS value of the total current of the combination is strongly reduced with respect to that of the LED6, mostly due to a decrease in the content of the third-order harmonic current, although some reduction is also observed for the high-order harmonics. It is important to note that the current of the LED6 has a strong component of the third-order harmonic (exceeding the emission limit imposed by IEC as quoted in Section 1) and also of high-order harmonics ( $h=9$ and 15). As the overall harmonic power losses in the neutral conductor depend on the RMS value of the total current, a marked reduction in the harmonic losses with respect to that of the LED6 (and even with respect to the LED5) is expected for the tested lamps combination.

Notice also the negligible-small value of the first-order harmonic current in the neutral conductor due to the balanced loading of the cable. The small residuals observed are due in part to small asymmetries attributable to the constructive differences between the lamps tested.

The cable harmonic power losses can be approximately calculated by the following formula,

$$
P_{\text {loss }}=3 \sum_{h=1}^{19} I_{R M S}{ }^{2}(h) R_{a c}(h)+\sum_{h=1}^{19} I_{R M S N}{ }^{2}(h) R_{a c N}(h),(2)
$$

being the first term on the right-hand side of (2) the losses in the phase conductors, and the second term the losses in the neutral conductor. $\left(I_{\mathrm{RMS}}(h)\right.$ and $I_{\mathrm{RMS}}(h)$ are the RMS value of the harmonic current of order $h$ in the phase and neutral conductor, and $R_{\mathrm{ac}}(h)$ and $R_{\mathrm{ac}} \mathrm{N}(h)$ are the ac resistance of the phase and neutral conductors at the frequency of the harmonic of order $h$, respectively).

To quantify the reduction in the harmonic power losses due to the lamps combination, it is useful to compare the above calculated cable losses (2) with the losses produced in an identical cable but carrying an undistorted electric current of the same RMS value as the first harmonic current of the distorted current. To do this, the cable losses ratio defined as,

$$
\xi \equiv \frac{P_{l o s s}}{3 I_{R M S}{ }^{2}(1) R_{a c}(1)+I_{R M S N}{ }^{2}(1) R_{a c N}(1)},
$$

(the first-order harmonic current in the neutral conductor is due to small asymmetries in the single-phase loads) was calculated on the basis of the measured data by neglecting the influence of the harmonic frequency on the resistance of the conductors (i.e., the conductor radius is small as compared to the characteristic skin penetration length and the distances of the nearby conductors are large as compared to the conductor radius [4]). For a neutral conductor having a cross-section equal to the half of the phase conductors section [13], it results in a value of 9.6, 3.3 and 2.8 for the individual lamps LED6 and LED 5, and for the combination; respectively, thus showing that the tested lamps combination leads to a significant decrease in the power harmonic losses. A similar result can be obtained for other lamps combination provided that the diversity factor for the third-order harmonic current of the arrangement is small enough.

Note that in lighting loads of substantial power demand such as those considered in this work, it would be convenient from the point of view of the reduction of the power losses, to connect the LED lamps between lines (rather than between a line and the neutral conductor). In such a case the third-order harmonic currents (and their multiples) cannot flow through the network since the return path through the neutral conductor does not exist. This suggests the convenience of having LED lamps including ac/dc converters designed to operate as two-phase loads. As quoted before, a large number of the existing low-voltage installations present a neutral conductor with a reduced section (about half of the phase conductor) [13]. These installations when feeding LED loads could present more than twice the losses corresponding to a current without distortion of the same RMS as the value of the first harmonic current of the lamps [4]; thus, a marked reduction (over $\sim 50 \%$ ) in the overall harmonic power losses can be expected if the LED lamps (having ac/dc converters designed to operate as two-phase loads) are connected between lines instead as single-phase loads.

\section{Conclusions}

An experimental investigation of diversity factors for LED (light emitting diode) in combination with CFL (compact fluorescent lamps) and LED lamps with nominal powers $<25 \mathrm{~W}$ was presented in this paper. In contrast to other works, attention was paid to the reduction of loworder harmonics, especially the third one; which is mainly responsible for the strong increase in power losses in the neutral conductor of the low-voltage installations. The results showed that a number of selected combination of LED and CFL lamps lead to a considerable decrease in the 
content of the third-order harmonic current. These results are different from those reported by other studies where lower order harmonics did not exhibit a very large reduction in amplitude. However, it should be noted that in those studies random combinations of lamps were used. The convenience of having LED lamps designed to operate as two-phase loads is suggested for certain applications of significant power demand.

\section{Acknowledgements}

L. P. and M. A. L. are members of the CONICET. N. M. and L. P. acknowledge financial support by Universidad Tecnológica Nacional (PID 3568).

\section{References}

[1] I. L. Azevedo, M. G. Morgan, F. Morgan, The transition to solid-state lighting, Proc. IEEE 97: 481510, 2009.

[2] S. Chong-Tan, General $n$-Level Driving Approach for Improving Electrical-to-Optical Energy-Conversion Efficiency of Fast-Response Saturable Lighting Devices, IEEE Trans. Ind. Electron. 57: 1342-1353, 2010.

[3] H. Chiung-Wen, Ch. Pao-Long, Sh. Yen-Hsun, Evaluating Government Policy on Accelerating the Use of LED Lighting Products Using System Dynamics Modeling in Taiwan, Proc. IEEE of PICMET '12: 50-59, 2012.

[4] N. Milardovich, L. Prevosto, M. A. Lara, Calculation of harmonic losses and ampacity in low-voltage power cables when used for feeding large LED lighting loads, Advanced Electromagnetics 3: 51-56, 2014.

[5] A. Dolara, S. Leva, Power Quality and Harmonic Analysis of End User Devices, Energies 5: 5453-5466, 2012.

[6] W. Chen, S. Y. Ron Hui, Elimination of an Electrolytic Capacitor in AC/DC Light-Emitting Diode (LED) Driver with High Input Power Factor and Constant Output Current, IEEE Trans. Power Electron. 27: 1598-1607, 2012.

[7] X. Qu, S. C. Wong, C. K. Tse, Resonance assisted buck converter for offline driving of power LED replacement lamps, IEEE Trans.Power Electron. 26: 532-540, 2011.

[8] S. Cha, D. Park, Y. Lee, Ch. Lee, J. Choi, J. Lee and H. Lee, AC/DC Converter Free LED Driver for Lightings, IEEE International Conference on Consumer Electronics (ICCE): 706-708, 2012.

[9] J. J. M. Desmet, I. Sweertvaegher, G. Vanalme, K. Stockman, R. J. M. Belmans, Analysis of the neutral conductor current in a three-phase supplied network with nonlinear single-phase loads, IEEE Trans. Ind. Appl. 39: 587-593, 2003.

[10] A. P. S. Meliopoulos, M. A. Martin Jr., Calculation of Secondary Cable Losses and Ampacity in the Presence of Harmonics, IEEE Trans. Power Delivery 7: 451-459, 1992.
[11]C. Demoulias, D. P. Labridis, P. S. Dokopoulos, K. Gouramanis, Ampacity of Low-Voltage Power Cables Under Nonsinusoidal Currents, IEEE Trans. Power Delivery 22: 584-594, 2007.

[12] V. Ćuk, J. F. G. Cobben, W. L. Kling, R. B. Timens, An analysis of diversity factors applied to harmonic emission limits for energy saving lamps, Proc. 14th International Conference on Harmonics and Quality of Power (ICHQP): Bergamo, Italy, 1-6, 2010.

[13] Distribution cables of rated voltage $0,6 / 1 \mathrm{kV}$, CENELEC Std. HD603 S1:1994/A2: 2003 E.

[14] M. Hongbo, G. Chen, J. Yi, Q. Meng, D. Sha, A Universal-Input Single-stage AC-DC Converter for TwinBus Type High-Power LED applications Energy Conversion Congress and Exposition (ECCE) IEEE: 1-6, 2016.

[15] K. Naraharisetti, P. B. Green, Primary Side Regulated Flyback AC-DC Converter for LED's International Conference on Electro/Information Technology (EIT) IEEE: 117-121, 2015

[16] S. Muthu, F.J. Schuunnans and M. Pashley, Red, green, and blue LEDs for white light illumination, IEEE J. Quantum Electron. 8: 333-338, 2002.

[17] S. Uddin, H. Shareef, A. Mohamed, M. A. Hannan, An Analysis of Harmonic Diversity Factors Applied to LED Lamps, Proc. POWERCON12, Auckland, New Zealand: 1-5, 2012. 